\title{
Analytical calculation of chain length in ferrofluids
}

\author{
M DEVI $^{1, *}$, P P DUTTA $^{2}$ and D MOHANTA ${ }^{1}$ \\ ${ }^{1}$ Nanoscience and Soft Matter Laboratory, Department of Physics, Tezpur University, Assam 784028, India \\ ${ }^{2}$ Department of Mechanical Engineering, Tezpur University, Assam 784028, India
}

MS received 4 November 2013; revised 21 March 2014

\begin{abstract}
The response of a typical ferrofluid (FF) lies in its explicit property of chain formation of magnetic nanoparticles. The most significant magneto-optic (MO) and magneto-viscous (MV) effects of FF are attributed to chaining effect. In the present research, an effort was made to analytically justify the dependence of the structure evolution of FFs on different measurable parameters involved in MO and MV effects. The problem is treated with the help of dimensional analysis and an empirical relation is formulated relating the equilibrium chain length with Verdet coefficient (constant), particle diameter, viscosity of the carrier fluid, particle density, magnetization and shear rate. The formulated relation of chain length is supported by error analysis to yield the uncertainty in the result. The maximum uncertainty in four sets of data is found as $\sim 0.75$.
\end{abstract}

Keywords. Ferrofluid; chain length; dimensional analysis; uncertainty analysis.

\section{Introduction}

In recent decades, ferrofluids (FFs) have emerged as an important topic both in the field of experimental and theoretical soft-matter research. A typical FF or magnetic fluid is a colloidal solution of magnetic nanoparticles (MNPs). ${ }^{1}$ Owing to their unusual particle-chain formation capability (figure 1), ${ }^{2,3}$ FFs exhibit several technologically important properties including magneto-optic (MO) and magnetoviscous (MV) responses). ${ }^{4-7}$ The underlying mechanism of chain formation, kinetics as well as the dynamics of these chains continues to be an interesting problem since the discovery of FFs till date. In the past, the evidence of field-induced aggregation/structure of FFs was observed in many studies, e.g., dichroism, ${ }^{8}$ Raman scattering, ${ }^{9}$ electron microscopy,${ }_{10}^{10}$ small-angle neutron scattering, ${ }^{11}$ atomic force microscopy, ${ }^{12}$ light scattering, ${ }^{13}$ etc. Popplewell et $a l^{14}$ have observed a chain-like structure in tin and copper FFs while studying the dichroism in the microwave region. They presented an image of linear arrangement of particles under an external magnetic field applied parallel to the slit containing the particles. ${ }^{14}$ Some better attestation of magnetic field-induced structural evolution of $\mathrm{Fe}_{3} \mathrm{O}_{4}$ nanoparticle-based FF emulsion was also presented in another report. ${ }^{15}$ Recently, a detailed structural evolution of the dispersed particles of an FF system was demonstrated through a precise light scattering study ${ }^{16}$ in which time-dependent intensity and scattered pattern of the particles were recorded. The variation of intensity and

\footnotetext{
*Author for correspondence (manasi@ tezu.ernet.in)
}

pattern formation by the scattered light indicated several critical fields and zippering effect of chains was attributed to these patterns. The phase diagram of chaining effect is also regarded as an important topic in many theoretical and simulation works. Long back, Krueger ${ }^{17}$ made an attempt to estimate the equilibrium chain lengths in magnetic colloids. Assuming the grains of identical size, the average number of grains per agglomerate was calculated in that report. A similar effort was also made in a recent study of FFs with large but finite aggregates of magnetic dipoles. ${ }^{18}$ It was noticed that with increase of size, the aggregates form chains and rings to multi-chains and multi-rings due to the competition among various energy terms. In fact, the chain-like structures come out as a result of competition between magnetic dipolar interaction and thermal interaction. The effective interaction between two ferromagnetic particles is generally expressed by a coupling constant given by Laskar et al: ${ }^{16}$

$$
\Lambda=\frac{\pi \mu_{o} \chi^{2} H^{2} D^{3}}{72 k_{\beta} T} .
$$

Here, $D$ is the diameter of the MNPs and $\chi$ is the effective susceptibility of individual MNP and $H$ is the applied magnetic field. The favourable condition for chain formation of the dispersed particles is $\Lambda \gg 1$. Again, reports show that different measurable parameters related with MO (e.g., applied wavelength) and MV (e.g., shear rate) response also play a crucial role in the process of structure evolution of FF. ${ }^{19}$ Unfortunately, the structural transition of magnetic fluid/FF remained a challenging task owing to the serious difficulties arising from the poly-disperse and dynamic 


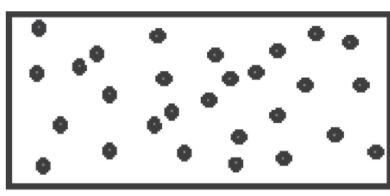

$B=0$
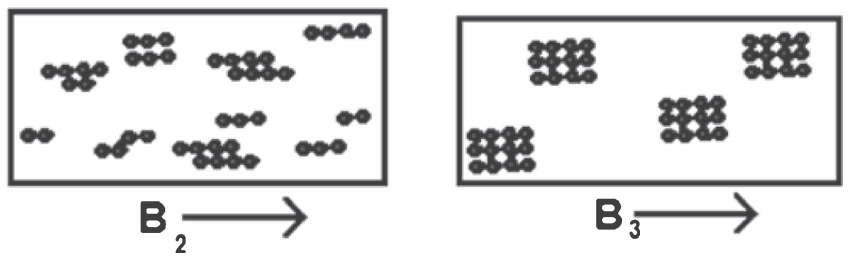

Figure 1. Schematic of structural evolution in FFs with increasing magnetic field from $\mathrm{B}=0$ to $\mathrm{B}_{3}$.

nature of the fluid. For the sake of convenience and ease of modelling, only mono-disperse or bi-disperse cases are generally considered.

To best of our knowledge, no result has been reported yet on chain length in a FF in terms of parameters related with $\mathrm{MO}$ and MV effect. Here, a technique is endeavoured in perspective of estimation of saturated chain length in typical FFs. Considering theories of fluid mechanics, present work associate with MO/MV effects analytically so as to correlate the chain length with different physical parameters. The respective parameters have been obtained from the Faraday rotation (FR) response and/or MV effect studied earlier at a select wavelength and moderate shear flow rate.

\section{Dimensional analysis}

Dimensional analysis is a widely employed technique in science. It is used for design, ordering and performance of model tests. More precisely, dimensional analysis can validate the relationship among different physical quantities through their dimensions. ${ }^{20}$ It is a better tool for complete understanding of a problem. The dimensional analysis can also be used to accumulate the results of experiments in a concise, easily reached form so that the broadly applicable general form can be attained from a small test. It is a general process of removal of units which ultimately removes the conversion factors.

If the number of variables associated with a physical phenomenon is known, then dimensional analysis can be done with the help of two methods: Rayleigh's method and Buckingham's $\pi$-theorem. ${ }^{21}$ The latter one is an extensively used theorem in dimensional analysis to solve a problem with number of variables. It gives a relation between a function expressed in terms of dimensional parameters and a related function in terms of non-dimensional parameters. Buckingham's $\pi$-theorem states, that in any physical problem, where ' $q$ ' quantities (e.g., velocity, pressure, etc.) involving ' $d$ ' basic dimensions (e.g., mass, time, length, etc.) were required to describe the problem, these quantities can be rearranged into at most $(q-d)$ independent dimensionless parameters. ${ }^{21}$ These dimensionless terms are called $\pi$-terms.

Mathematically, if a variable $X_{1}$ depends on independent variables $X_{2}, X_{3}, X_{4} \ldots \ldots X_{n}$, then $X_{1}$ can be written as:

$$
X_{1}=f\left(X_{2}, X_{3}, X_{4} \ldots X_{n}\right) .
$$

Or

$$
f_{1}\left(X_{1}, X_{2}, X_{3}, X_{4} \ldots X_{n}\right)=0 .
$$

If there are $m$ fundamental dimensions, then according to the Buckingham's $\pi$-theorem eq. (3) can be written in terms of $\pi$-terms, in which a number of $\pi$-terms is equal to $(n-m)$. So, eq. (3) becomes

$$
f_{1}\left(\pi_{1}, \pi_{2}, \pi_{3}, \ldots \pi_{n-m}\right)=0 .
$$

Each $\pi$-term contains $(m+1)$ variables. The $m$ variables that appear repeatedly in each $\pi$-term are called repeating variables. The repeating variables are such that, they themselves must not form any dimensionless quantity and must contain jointly all the fundamental dimensions present in the problem. If $X_{2}, X_{3}$ and $X_{4}$ are considered as repeating variables and if there are three fundamental dimensions (say, $M, L$ and $T$ ), then each $\pi$-term will be in the form:

$$
\begin{aligned}
\pi_{1} & =X_{2}^{a_{1}} \cdot X_{3}^{b_{1}} \cdot X_{4}^{c_{1}} \cdot X_{1} \\
\pi_{2} & =X_{2}^{a_{2}} \cdot X_{3}^{b_{2}} \cdot X_{4}^{c_{2}} \cdot X_{5} \\
\vdots & \\
\pi_{n-m} & =X_{2}^{a_{n-m}} \cdot X_{3}^{b_{n-m}} \cdot X_{4}^{c_{n-m}} \cdot X_{n}
\end{aligned}
$$

where $a_{1}, b_{1}, c_{1} ; a_{2}, b_{2}, c_{2}$, etc. are constants. These constants are determined by considering dimensional homogeneity. The final general equation for the phenomenon may be obtained as follows,

$$
\begin{aligned}
\pi_{1} & =\varphi\left(\pi_{2}, \pi_{3}, \pi_{4} \ldots \ldots \pi_{n-m}\right) \\
\pi_{2} & =\varphi\left(\pi_{1}, \pi_{3}, \pi_{4} \ldots \ldots \pi_{n-m}\right)
\end{aligned}
$$

\section{Error and uncertainty analysis}

Error and uncertainty always creep into all experimental data in spite of adequate care being taken in the calibration and data acquisition process. While performing an experiment, errors can occur due to various reasons, viz. the faulty design of the experimental setup, insufficient data, lack of calibration, etc. The errors can be of different origin and nature, viz. random errors, fixed errors (appear in repeated reading by the same amount but for unknown reasons), etc. ${ }^{22}$ Analysis must be performed on all experimental results to ease out uncertainty and minimise errors. The accuracy of an experiment is limited unless it is scrutinized by error analysis. 
The errors in the experimental data lead to some extent of uncertainty in the results. Thus, the amount of uncertainty has to be calculated in a definite way. Based on specification of the uncertainty in the various primary experimental measurements, Kline and $\mathrm{McClintok}^{23}$ have proposed a method to estimate the uncertainty in the experimental results, given by:

$$
W_{r}=\left[\left(\frac{\partial R}{\partial x_{1}} W_{1}\right)^{2}+\left(\frac{\partial R}{\partial x_{2}} W_{2}\right)^{2}+\cdots \cdots \cdots\right]^{1 / 2}
$$

Here, $R$ is the experimental outcome and function of independent variables $x_{1}, x_{2}, x_{3} \ldots$, the uncertainty in the result is $W_{r}$ and those in the independent variables are $W_{1}, W_{2}, W_{3} \ldots \ldots$

\section{Analytical calculation of equilibrium chain length in $\mathbf{F F}$}

\subsection{Dimensional technique and the FF systems}

The chaining effect plays an important role in the modified MO responses of FFs and earlier, it was shown that FR has a dependency on the chain length. ${ }^{24,25}$ Eventually, the Verdet coefficient (constant) was also found to be influenced due to the variation in the chain length. ${ }^{26}$ Typically, the FR first increases with field and then tends to saturate. The saturation indicates the limit of a chain. Zippering or bundle of chains starts from the saturation point. Again, eq. (1) shows that the chaining effect is expected to be controlled by the particle size, magnetization and other parameters (coupling constant).

The viscosity of the carrier fluid of a FF certainly matters since, with the increase of the viscosity of the carrier fluid, the FR response drops substantially. In a more viscous fluid, the viscous force acts as a hindrance to the chain formation resulting in a suppression of the FR and the Verdet coefficient. The FR was also shown to be dependent on the concentration of the FF where the chain length at a given field is found to be linear with concentration. ${ }^{27}$ The concentration of any FF, expressed in terms of $\mathrm{v} / \mathrm{v}$ or $\mathrm{wt} / \mathrm{v}$, mainly signifies the density of the dispersed particles with surfactant coating.

In a FF, the dispersed nanoparticles experience both translational and rotational motion. The particles, under an external field, endure magnetic force-driven diffusion process. The overall change of the particle concentration is more important than the individual velocity and trajectory of the particle in such cases. In a FF flow, the velocity gradient plays a crucial role in chaining effect. The gradient of velocity in any flowing material can be expressed by the shear rate, which has a significant influence on the particles' chain in the FF. ${ }^{28}$ With increasing shear rate, the agglomeration or the chains break down resulting in non Newtonian nature of the FF. Thus, the transformation of the MO property of FF with shear rate is much expected like MV effect.
On the basis of the above discussion, an attempt was made in the present research to find an empirical correlation of the FR response in terms of different factors. For the sake of convenience of using Buckingham's $\pi$-theorem, the Verdet coefficient, rather than the rotation itself, was considered. It may be noted that the Verdet coefficient $(V)$ of a FF is a function of chain length $(l)$, particle diameter $(D)$, viscosity of the carrier fluid $(\eta)$, particle density $(\rho)$, magnetization $(m)$ and shear rate $(\dot{\gamma})$.

Mathematically, it can be expressed as:

$$
V=f\left(l, D, \eta^{-1}, \rho, m, \dot{\gamma}\right) .
$$

Or

$$
f_{1}\left(V, l, D, \eta^{-1}, \rho, m, \dot{\gamma}\right)=0 .
$$

Thus, the total number of variables $=7$. In order to predict the total number of fundamental dimensions, we express each of the variables in terms of its dimension as follows,

$$
\begin{array}{ll}
V=M^{-1} L^{-1} Q(\mathrm{rad} / \mathrm{T}-\mathrm{m}) & l=L(\mathrm{~m}) \\
D=L(\mathrm{~m}) & \eta^{-1}=M^{-1} L T(\mathrm{~Pa} . \mathrm{s})^{-1} \\
\rho=M L^{-3}\left(\mathrm{~kg} \mathrm{~m}^{-3}\right) & m=L^{-1} T^{-1} Q\left(\mathrm{~A} \mathrm{~m}^{-1}\right) \\
\dot{\gamma}=T^{-1}\left(\mathrm{~s}^{-1}\right) &
\end{array}
$$

The total number of fundamental dimensions $=4(M, L, T$ and $Q$ ).

According to Buckingham's $\pi$-theorem, total number of $\pi$ terms $=3$. Consequently, three $\pi$-terms (say, $\pi_{1}, \pi_{2}$ and $\pi_{3}$ ) are formed. Now, eq. (7) can be rewritten as:

$$
f_{1}\left(\pi_{1}, \pi_{2}, \pi_{3}\right)=0
$$

\subsection{Selection of repeating variables}

In this problem, the total number of fundamental dimensions is 4 . Therefore, out of the seven quantities $(V, l, d$, $\left.\mu^{-1}, \rho, m, u\right)$, four variables must be chosen as repeating variables. Being the dependent variable, $V$ is excluded from being selected as a repeating variable. We consider $l, \quad \rho, \quad m, \dot{\gamma}$ as repeating variables, as the fundamental dimensions $M, L, T$ and $Q$ are present in them and they do not form any dimensionless term.

Table 1. Different FFs used in this calculation. ${ }^{26,31}$

\begin{tabular}{lccc}
\hline Ferrofluid & Magnetic particles & Surfactant & Carrier fluid \\
\hline FFW & $\mathrm{Fe}_{3} \mathrm{O}_{4}$ & Oleic acid & Milli-Q-water \\
FFK & $\mathrm{Fe}_{3} \mathrm{O}_{4}$ & Oleic acid & Kerosene \\
FF1 & $\mathrm{Fe}_{3} \mathrm{O}_{4}$ & $\begin{array}{c}\text { Tetra-methyl ammonium } \\
\text { hydroxide (TMAH) }\end{array}$ & $\begin{array}{c}\text { Methanol } \\
\text { FF2 }\end{array}$ \\
$\mathrm{Fe}_{3} \mathrm{O}_{4}$ & Oleic acid & Methanol \\
\hline
\end{tabular}


Table 2. Different parameters of the FFs obtained from experimental results. ${ }^{26,31}$

\begin{tabular}{llclrr}
\hline Ferrofluid & $V(\mathrm{rad} / \mathrm{T}-\mathrm{m})$ & $\dot{\gamma}\left(\mathrm{s}^{-1}\right)$ & $\eta(\mathrm{Pa}-\mathrm{s})$ & $m\left(\mathrm{~A} \mathrm{~m}^{-1}\right)$ & $D(\mathrm{~m})$ \\
\hline FFW & $0.11 \times 10^{3}$ & 4 & 0.0073 & 3529 & $8 \times 10^{-9}$ \\
FFK & $0.081 \times 10^{3}$ & 5.1 & 0.0082 & 3508 & $10 \times 10^{-9}$ \\
FF1 & $0.05 \times 10^{3}$ & 44.3 & 0.009 & 2400 & $12 \times 10^{-9}$ \\
FF2 & $0.052 \times 10^{3}$ & 22.8 & 0.013 & 2400 & $12 \times 10^{-9}$ \\
\hline
\end{tabular}

Table 3. Different initial parameters.

\begin{tabular}{lclcrr}
\hline Ferrofluid & $\dot{\gamma}\left(\mathrm{s}^{-1}\right)$ & $\eta(\mathrm{Pa}-\mathrm{s})$ & $m\left(\mathrm{~A} \mathrm{~m}^{-1}\right)$ & $D(\mathrm{~m})$ & $\rho\left(\mathrm{kg} \mathrm{m}^{-3}\right)$ \\
\hline FFW & 90 & 0.005 & 3529 & $8 \times 10^{-9}$ & 0.175 \\
FFK & 110 & 0.0059 & 3508 & $10 \times 10^{-9}$ & 0.175 \\
FF1 & 100 & 0.0064 & 2400 & $12 \times 10^{-9}$ & 0.66 \\
FF2 & 105 & 0.011 & 2400 & $12 \times 10^{-9}$ & 0.48 \\
\hline
\end{tabular}

Table 4. Calculated chain length for different ferrofluids.

\begin{tabular}{lc}
\hline Ferrofluid & Chain length $(\mu \mathrm{m})$ \\
\hline FFW & 400 \\
FFK & 500 \\
FF1 & 600 \\
FF2 & 700 \\
\hline
\end{tabular}

Now, the three $\pi$-terms are selected as follows:

$$
\begin{aligned}
& \pi_{1}=l^{a_{1}} \dot{\gamma}^{b_{1}} \rho^{c_{1}} m^{d_{1}} V \\
& \pi_{2}=l^{a_{2}} \dot{\gamma}^{b_{2}} \rho^{c_{2}} m^{d_{2}} \eta^{-1} \\
& \pi_{3}=l^{a_{3}} \dot{\gamma}^{b_{3}} \rho^{c_{3}} m^{d_{3}} D
\end{aligned}
$$

Each $\pi$-term is solved by the principle of dimensional homogeneity as follows:

\section{$\pi_{1}$-term}

$M^{\circ} L^{\circ} T^{\circ} Q^{\circ}=L^{a_{1}} \cdot\left(T^{-1}\right)^{b_{1}} \cdot\left(M L^{-3}\right)^{c_{1}} \cdot\left(L^{-1} T^{-1} Q\right)^{d_{1}} \cdot\left(M^{-1} L^{-1} Q\right)$

Comparing the exponents of $M, L, T$ and $Q$, respectively, $a_{1}=3, b_{1}=1, c_{1}=1$ and $d_{1}=-1$

$$
\therefore \pi_{1}=l^{3} \dot{\gamma} \rho m^{-1} V
$$

\section{$\pi_{2}$-term}

$M^{\circ} L^{\circ} T^{\circ} Q^{\circ}=L^{a_{2}} \cdot\left(T^{-1}\right)^{b_{2}} \cdot\left(M L^{-3}\right)^{c_{2}} \cdot\left(L^{-1} T^{-1} Q\right)^{d_{2}} \cdot\left(M^{-1} L T\right)$

Comparing the exponents of $M, L, T$ and $Q$, respectively, $a_{2}=2, b_{2}=1, c_{2}=1$ and $d_{2}=0$

$$
\therefore \pi_{2}=l^{2} \dot{\gamma} \rho \eta^{-1}
$$

\section{$\pi_{3}$-term}

$M^{\circ} L^{\circ} T^{\circ} Q^{\circ}=L^{a_{3}} \cdot\left(T^{-1}\right)^{b_{3}} \cdot\left(M L^{-3}\right)^{c_{3}} \cdot\left(L^{-1} T^{-1} Q\right)^{d_{3}} \cdot L$
Comparing the exponents of $M, L, T$ and $Q$, respectively, $a_{3}=-1, b_{3}=0, c_{3}=0$ and $d_{3}=0$.

$$
\therefore \pi_{3}=l^{-1} D
$$

Substituting the value of $\pi_{1}, \pi_{2}$ and $\pi_{3}$ in eq. (7)

$$
f_{1}\left(l^{3} \dot{\gamma} \rho m^{-1} V, l^{2} \dot{\gamma} \rho \eta^{-1}, l^{-1} D\right)=0
$$

Or

$$
l^{3} \dot{\gamma} \rho m^{-1} V=c\left(l^{2} \dot{\gamma} \rho \eta^{-1}\right)^{p}\left(l^{-1} D\right)^{q}
$$

Here, $c$ is a material-specific constant. Now, taking ln on both the sides,

$$
\begin{aligned}
& \ln \left(l^{3} \dot{\gamma} \rho m^{-1} V\right)=\ln \left[c\left(l^{2} \dot{\gamma} \rho \eta^{-1}\right)^{p}\left(l^{-1} D\right)^{q}\right] \\
& (3-2 p+q) \ln l=\ln c+(p-1) \ln \dot{\gamma} \\
& +(p-1) \ln \rho-p \ln \eta+\ln m-\ln V+q \ln D .
\end{aligned}
$$

The $p$ and $q$ values were determined with the help of the experimental results. Here, four synthesized FFs are considered, namely, FFW, FFK, FF1 and FF2 (table 1).

The values of different parameters corresponding to a field $\sim 100 \mathrm{G}$ used in this calculation are highlighted in table 2 .

Using eq. (12) for different sets of data as shown in table 2, the values of $p$ and $q$ are found to be $\sim 0.67$ and $\sim 2$. Putting the values of $p$ and $q$ in eq. (12)

$$
\begin{aligned}
3.7 \ln l= & \ln c-0.3 \ln \dot{\gamma}-0.31 \ln \rho-0.68 \ln \eta \\
& +\ln m-\ln V+2 \ln D .
\end{aligned}
$$

Or

$$
\begin{gathered}
\ln l=0.27 \ln c-0.09 \ln \dot{\gamma}-0.09 \ln \rho-0.18 \ln \eta \\
+0.27 \ln m-0.27 \ln V+0.56 \ln D .
\end{gathered}
$$

Eq. (14) gives an empirical formula for obtaining chain length of nanoparticles in a given FF. The values of all the 
Table 5. Error analysis and overall uncertainty.

\begin{tabular}{|c|c|c|c|c|c|c|}
\hline Ferrofluid & $V(\operatorname{rad} / \mathrm{T}-\mathrm{m})$ & $\eta(\mathrm{Pa}-\mathrm{s})$ & $m\left(\mathrm{~A} \mathrm{~m}^{-1}\right)$ & $D(\mathrm{~m})$ & $\rho\left(\mathrm{kg} \mathrm{m}^{-1}\right)$ & $W_{r}$ \\
\hline FFW & \pm 0.0014 & \pm 0.05 & \pm 3 & $\pm 2 \times 10^{-9}$ & \pm 0.007 & $\sim 0.5$ \\
\hline FFK & \pm 0.0018 & \pm 0.0821 & \pm 1 & $\pm 2 \times 10^{-9}$ & \pm 0.007 & $\sim 0.75$ \\
\hline FF1 & \pm 0.0098 & \pm 0.0637 & \pm 2 & $\pm 2 \times 10^{-9}$ & \pm 0.002 & $\sim 0.54$ \\
\hline FF2 & \pm 0.0052 & \pm 0.03 & \pm 2 & $\pm 2 \times 10^{-9}$ & \pm 0.002 & $\sim 0.002$ \\
\hline
\end{tabular}

variables/measurable parameters in the above equation can be evaluated experimentally. The value of the constant $c$ is determined from the initial condition that, in the absence of an applied magnetic field, the particles are in random motion. In that case, the length of the field-induced chain is considered as zero. Consequently, the value of the Verdet coefficient is also discarded. Under high shear rate condition, the few pre-existing aggregates break down resulting in a uniformly dispersed FF with comparatively low viscosity. The initial parameters corresponding to zero chain length and calculated value of the constant $c$ for each data set are shown in table 3 .

Finally, the predicted chain lengths of different FFs for a static field strength $\sim 100 \mathrm{G}$ are shown in table 4 .

It was found that, the MNPs of the FFs are capable of forming chains of length in the micrometre range when subjected to an applied field of $\sim 100 \mathrm{G}$. This is supported by an earlier report that, a $\mathrm{Fe}_{3} \mathrm{O}_{4}$ magnetic fluid with polymer acrylic resin forms chains of length in the micrometre scale $(>200 \mu \mathrm{m})$ in response to a moderate magnetic field strength (281-1030) Oe. ${ }^{29}$ With reference to eq. (1) it can be said that the bigger particles have a better chain-forming capability than the smaller ones. The calculated smaller chain length in FFW than in FFK is mainly attributed to the smaller particle size in the former FF. Again, the dispersion stability of surfactant depends on the hydrocarbon chain length. Due to the longer hydrocarbon chain, the absorption-free energy in TMAH (surfactant of FF1) is higher than that of oleic acid (surfactant of FF2). ${ }^{30}$ Consequently, the chaining effect can be adequately suppressed owing to better chemical stability of FF1 over its FF2 counterpart. In addition, a larger value of the shear rate in FF1 in the calculation part could also contribute to the shorter chain length. Not surprisingly, the chains or aligned aggregates could break down at an increased shear rate.

\subsection{Uncertainty calculation}

The uncertainty in the above calculation is determined by the following method as described in section 2 . The uncertainty in each data set is calculated by eq. (4). The errors in individual data and overall uncertainty corresponding to the equilibrium chain length of a FF are described in table 5.

\section{Conclusions}

The dimensional analysis of the Verdet coefficient (constant) and its dependency on various parameters, following
Buckingham's $\pi$-theorem, gives an empirical formula to calculate the equilibrium chain length of the magnetite-based FFs used in other experiments. It is calculated for four different FF systems with varying MO and MV conditions. The chain length for all the four FFs is found in the micrometre range with nominal uncertainty. A more careful investigation through computer simulation is in progress.

\section{References}

1. Papell S S 1964 US Pat. No. 3215572

2. Odenbach S (Ed) 2009 Colloidal magnetic fluids (Berlin Heidelberg: Springer)

3. Zubarev A Y and Iskakova L Y 2002 Phys. Rev. E 65061406

4. Ghasemi E, Mirhabibi A and Edrissi M 2011 J. Nanosci. Nanotech. 115285

5. Odenbach S 2002 Magnetoviscous effects in ferro fluids (Berlin Heidelberg: Springer)

6. Paul N, Devi M, Mohanta D and Saha A 2012 J. Appl. Phys. 11104490

7. Taketomi S, Ukita M, Mizukami M, Miyajima $\mathrm{H}$ and Chikazumi S 1987 J. Phy. Sos. Japn. 563362

8. Davies H W and Llewellyn J P 1980 J. Phys. D: Appl. Phys. 132327

9. Mendelev V S and Ivanov A O 2004 Phys. Rev. E 70 051502

10. Butter K, Bomans P H H, Frederik P M, Vroege G J and Philipse A P 2003 Nat. Mater. 288

11. Pyanzina E, Kantorovich S, Cerdà J J and Holm C $2011 \mathrm{~J}$. Magn. Magn. Mater. 3231263

12. Groß C, Büscher K, Romanus E, Helm C A and Weitschies W 2002 Euro. Cell. Mater. 3163

13. Laskar J M, Brojabasi S, Raj B and Philip 2012 J. Opt. Commun. 285242

14. Popplewell J, Davies P, Llewellyn J P and O'grady K 1986 J. Magn. Magn. Mater. 54-57 761

15. Ivery M, Liu J, Zhu Y and Cutillas S 2003 Phys. Rev. E 63 011403

16. Laskar J M, Philip J and Raj B 2009 Phys. Rev. E 80041401

17. Krueger D A 1979 J. Coll. Inter. Sci. 70558

18. Yoon M and Tomanek D 2010 J. Phys.: Condens. Matter. 22 455105

19. Pop L M, Odenbach S A, Wiedenmaan N M and Bönnemann H 2005 J. Magnet. Magnet. Mater. 289303

20. Palmer A C 2008 Dimensional analysis and intelligent experimentation (Singapore: World Scientific Publishing)

21. Graebel W P 2001 Engineering fluid mechanics (New York: Taylor and Francis) 
22. Holman J P 2007 Experimental methods for engineers (New Delhi: Tata McGraw Hill)

23. Kline S J and McClintok F A 1953 Mech. Eng. 753

24. Philip J and Laskar J M 2012 J. Nanofluid. 13

25. Rousan A A, Hassan E G and Yusuf N A 1989 IEEE Trans. Magn. 253121

26. Devi M, Das R, Mohanta D, Baruah K K and Saha A 2012 Appl. Phys. A 106765
27. Yusuf N A, Aljarayesh A I, Rousan A A and El-Ghanem H M 1990 IEEE Trans. Magn. 262852

28. Odenbach S and Störk H 1998 J. Magn. Magn. Mater. 183188

29. Fang W X, He Z H, Xu X Q, Mao Z Q and Shen H 2007 Euro. Phys. Lett. 7768004

30. Hetem M J J 1990 Anal. Chem. 622288

31. Singh H and Lipson R H (eds) 2009 Transport and optical properties of nanomaterials (New York: AIP Publishing) 495 